\title{
Comprehensive Analysis and Evaluation of Metro Transfer Time Perception
}

\author{
Weixin Hua ${ }^{1, \text { a }}$, Xuesong Feng ${ }^{1, b}$, Xiaojing Zhu ${ }^{1, c}$, and Yuanpeng Jie ${ }^{2, ~ d, ~ * ~}$ \\ ${ }^{1}$ School of Traffic and Transportation, Beijing Jiaotong University, Beijing 100044, China \\ ${ }^{2}$ China Railway Siyuan Survey and Design Group Co., LTD., Wuhan 430063, China

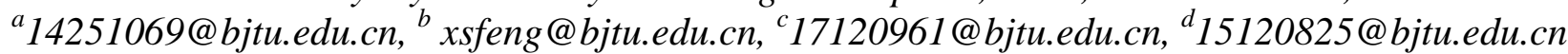

Keywords: Metro transfer time perception, Paired-samples T test, Ordinal logistic regression model, Influencing factors, Scenario analysis.

\begin{abstract}
With the development of rail transit network, the transfer between different subway lines has become an inevitable travel activity for many travelers. Considering the importance of passenger's transfer experience, this study selects the metro transfer time perception from the passenger's point of view as the research object. Based on the perceived and actual transfer data of Beijing subway passengers, the paired-samples $T$ test is used to verify the differences in passengers' perception of transfer. Then this study constructs the ordinal logistic regression model to analyze the influencing factors of transfer time perception, and explores ways to reduce it through scenario analysis. The results show that transfer passengers generally overestimate their transfer time and transfer distance. Additionally, the actual transfer waiting time is a key influencing factor because its reduction will significantly cause the decline in transfer time perception, especially when it is reduced to "3 minutes to 5 minutes".
\end{abstract}

\section{Introduction}

In recent years, with the development of rail transit, metro transfer time affecting passenger's transfer evaluation has become a key focus of many scholars while perceived transfer time may better reflect the passenger's real transfer experience compared with the actual transfer time. Current research on travel time perception is mainly focused on the travel time of the entire trip or other travel stage rather than the transfer stage, such as the waiting stage. Yu [1] and Delclòs-Alió et al. [2] argued the perceived differences in total travel time from the perspective of personal characteristics and travel characteristics, while Zhu [3] added external traffic environmental factors based on it. As to waiting time perception, Watkins et al. [4] and Fan et al. [5] studied the impact of mobile real-time information, the presence of infrastructure, and gender differences on it in unsafe environments. Additionally, some scholars further considered the interaction between perceived travel times of different travel phases [6, 7]. Therefore, based on the lack of existing research on the metro transfer time perception, this study will conduct a comprehensive analysis of its influencing factors and 
further explore ways to reduce it. This article is organized as follows. The survey data are analyzed in Section 2, followed by modeling of metro perceived transfer time in Section3. Next, Section 4 includes scenario analysis of various ways on reducing passengers' metro transfer time perception. The conclusions are given in Section 5.

\section{Data Survey}

This study combines the methods of questionnaire survey with field survey to obtain the perceived and actual data of metro transfer. Based on previous studies, this study designs questionnaire from three categories of variables including personal attributes, travel characteristics and factors related to metro transfer. After conducting the questionnaire survey to the transfer passengers of Beijing subway from November 29 to December 5, 2017, 490 questionnaires are returned and 467 are valid. Among the respondents, respondents aged 18 to 39 occupy $91.67 \%$ and $85.05 \%$ of respondents with monthly income below RMB 10,000, which is basically consistent with the characteristics of Beijing subway passengers. Based on the perceived and actual data of the transfer, the paired-sample $\mathrm{T}$ test is used to verify the passengers' perceived differences in transfer phase. The results show that the transfer passengers showed an overestimation of the perception of the walking time, walking distance and waiting time of the transfer. Additionally, compared to the waiting period ( 0.35 units), passengers overestimate the experience of the walking stage more severely (0.56 and 0.86 units).

Table 1 Paired-samples T test results of metro transfer perception differences

\begin{tabular}{lccccccc}
\hline $\begin{array}{l}\mathrm{N}=467 \\
\text { Paired differences }\end{array}$ & Mean & SD & \multicolumn{7}{c}{$\begin{array}{c}\text { 95\% Confidence interval of } \\
\text { the difference }\end{array}$} & T & Sig \\
\hline $\begin{array}{l}\text { perceived walking time-actual walking time } \\
\text { perceived walking distance-actual walking }\end{array}$ & 0.56 & 0.82 & 0.49 & 0.64 & 14.85 & 0.00 \\
distance & 0.86 & 1.47 & 0.73 & 0.99 & 12.63 & 0.00 \\
perceived waiting time-actual waiting time & 0.35 & 0.69 & 0.29 & 0.41 & 10.93 & 0.00 \\
\hline
\end{tabular}

\section{Modeling Study}

Considering the data format of metro transfer perception time of this study is discrete and orderly, the study will use the ordinal logistic regression model to analyze its influencing factors [8,9]. If the dependent variable includes k categories, and the model corresponds to k-1 formulas while the values of the independent variable parameters of these $k-1$ formulas are invariant except for the intercept parameters [10]. As to the explanation of parameter of independent variable, the Odds Ratio (OR) of each unit change of $x_{i}$ is $\exp \left(\beta_{i}\right)$ when other independent variables remain unchanged. The basic form of the model is as follows:

$$
\begin{aligned}
& \operatorname{Logit}(\Omega)=\ln \left(\frac{\gamma_{\mathrm{j}}}{1-\gamma_{\mathrm{j}}}\right)=\alpha_{\mathrm{j}}-\beta^{\prime} \mathrm{X}+\varepsilon, \mathrm{j} \text { includes } 1,2,3 \ldots \mathrm{k}-1 \\
& \gamma_{\mathrm{j}}=\frac{\exp \left(\alpha_{\mathrm{j}}-\beta^{\prime} \mathrm{X}+\varepsilon\right)}{1+\exp \left(\alpha_{\mathrm{j}}-\beta^{\prime} \mathrm{X}+\varepsilon\right)} \\
& \pi(\mathrm{X})=\mathrm{P}(\mathrm{y}=\mathrm{j} \mid \mathrm{X})=\gamma_{\mathrm{j}}-\gamma_{\mathrm{j}-1}
\end{aligned}
$$


Where $\Omega$ denotes Odds meaning the ratio of the probability of occurrence and non-occurrence of an event, $\gamma_{j}$ presents the cumulative probability of the first $\mathrm{j}$ categories of the dependent variable, $\mathrm{j}$ presents the category of the dependent variable, $\alpha_{\mathrm{j}}$ presents intercept parameter of the jth formula, $\beta^{\prime}$ presents parameter vector of independent variables, $\mathrm{X}$ presents the vector of independent variables and $\varepsilon$ denotes errors item.

\subsection{Metro Perceived Transfer Time Modelling}

Taking the three categories of influencing factors as independent variables, the metro perceived transfer time as dependent variable, an ordinal logistic regression model is constructed. Among them, there are four categories of the dependent variable, which is less than 5 minutes, 5 minutes to 10 minutes (exclusive), 10 minutes to 15 minutes (exclusive), and 15 minutes or more. After using the maximum likelihood estimation method to estimate the model, the estimation results are shown in table 2 below.

In the table 2, $\mathrm{XB}$ is $0-1$ variable, valuing 1.00 when passenger is men. $\mathrm{CT}_{1}$ and $\mathrm{CT}_{2}$ are $0-1$ variables, indicating whether the passenger's travel time is "less than one hour", "one hour to two hours (exclusive)". ZT presents perceived transfer walking time, including "less than 3 minutes", "3 minutes to 5 minutes(exclusive)", " 5 minutes to 10 minutes(exclusive)", "10 minutes or more”. SD, $\mathrm{SD}_{2}$ and $\mathrm{SD}_{3}$ are 0-1 variables, indicating whether the passenger's actual transfer walking distance is "less than 100 meters", "100 meters to 200 meters (exclusive)", "200 meters to 300 meters (exclusive)". $\mathrm{DT}_{1}$ and $\mathrm{DT}_{2}$ are $0-1$ variables, indicating whether the passenger's perceived transfer waiting time is "less than 3 minutes", " 3 minutes to 5 minutes (exclusive)". $\mathrm{LF}_{1}$ and $\mathrm{LF}_{2}$ are $0-1$ variables, indicating whether the number of stairs during the walking process is "less than 1", "2 to 3 (inclusive)". YJ presents the crowdedness of the walking process, including "uncongested", “congested (acceptable)", "very congested”.

Table 2 Estimation results of metro perceived transfer time model

\begin{tabular}{|c|c|c|c|c|c|c|}
\hline \multirow[t]{2}{*}{ Variable } & \multirow[t]{2}{*}{ Parameter } & \multirow[t]{2}{*}{ SD } & \multirow[t]{2}{*}{ Wald } & \multirow[t]{2}{*}{ Sig } & \multicolumn{2}{|c|}{$\begin{array}{c}\text { 95\% Confidence interval } \\
\text { of the difference }\end{array}$} \\
\hline & & & & & Upper & Lower \\
\hline$\alpha_{1}$ & -0.40 & 1.20 & 0.11 & 0.74 & -2.75 & 1.96 \\
\hline$\alpha_{2}$ & 2.57 & 1.21 & 4.54 & 0.03 & 0.21 & 4.94 \\
\hline$\alpha_{3}$ & 5.14 & 1.24 & 17.18 & 0.00 & 2.71 & 7.57 \\
\hline XB & -0.54 & 0.19 & 7.64 & 0.01 & -0.92 & -0.16 \\
\hline $\mathrm{CT}_{1}$ & -1.16 & 0.50 & 5.27 & 0.02 & -2.14 & -0.17 \\
\hline $\mathrm{CT}_{2}$ & -0.77 & 0.50 & 2.36 & 0.12 & -1.76 & 0.21 \\
\hline ZT & 0.77 & 0.33 & 5.31 & 0.02 & 0.11 & 1.42 \\
\hline $\mathrm{SD}_{1}$ & -1.10 & 0.46 & 5.86 & 0.02 & -1.99 & -0.21 \\
\hline $\mathrm{SD}_{2}$ & -0.95 & 0.44 & 4.75 & 0.03 & -1.80 & -0.10 \\
\hline $\mathrm{SD}_{3}$ & -0.25 & 0.43 & 0.34 & 0.56 & -1.08 & 0.59 \\
\hline $\mathrm{LF}_{1}$ & 2.59 & 1.04 & 6.24 & 0.01 & 0.56 & 4.62 \\
\hline $\mathrm{LF}_{2}$ & 2.93 & 0.79 & 13.75 & 0.00 & 1.38 & 4.48 \\
\hline YJ & 0.82 & 0.30 & 7.47 & 0.01 & 0.23 & 1.41 \\
\hline $\mathrm{DT}_{1}$ & -3.48 & 0.91 & 14.73 & 0.00 & -5.26 & -1.71 \\
\hline $\mathrm{DT}_{2}$ & -2.98 & 0.84 & 12.45 & 0.00 & -4.63 & -1.32 \\
\hline $\mathrm{DT}_{1} * \mathrm{ZT}$ & 1.23 & 0.40 & 9.30 & 0.00 & 0.44 & 2.02 \\
\hline $\mathrm{DT}_{2} * \mathrm{ZT}$ & 1.00 & 0.36 & 7.73 & 0.01 & 0.30 & 1.71 \\
\hline $\mathrm{LF}_{1} * \mathrm{YJ}$ & -0.83 & 0.44 & 3.63 & 0.06 & -1.69 & 0.02 \\
\hline $\mathrm{LF}_{2} * \mathrm{YJ}$ & -1.00 & 0.33 & 9.20 & 0.00 & -1.65 & -0.35 \\
\hline
\end{tabular}


From the table 2, the OR of gender is 0.58 , which indicates that the perceived transfer time of male is generally lower than of women. Moreover, the travel time is "less than one hour" with an OR of 0.31 , showing the transfer time perception of these passengers is generally lower than of passengers whose travel time is "two hours or more". In addition, the passenger's transfer time perception is also influenced by the actual and perceived situation of the walking stage and the perception of the waiting stage. As to cross terms, the joint contribution of the number of stairs and crowdedness of the walking process shows that the two form the walking environment of transfer passengers and then affect passengers' transfer experience. Additionally, perceived transfer walking time and waiting time both acts individually and jointly on the transfer time perception, indicating that passengers do not consider the feeling of a single transfer stage but the entire transfer process when they recall their transfer experiences.

\subsection{Metro Perceived Transfer Waiting Time Modelling}

In order to achieve an in-depth analysis of the transfer time perception, the following will further conduct an ordinal logistic regression model based on its important influencing factor - perceived transfer waiting time including three categories, which is less than 3 minutes, 3 minutes to 5 minutes (exclusive), and 5 minutes or more. The independent variables of this model are set as above. The estimation results of the model see below table 3, where ST presents actual transfer waiting time, whose values are the same as the perceived waiting time, $\mathrm{DR}_{1}$ and $\mathrm{DR}_{2}$ are $0-1$ variables, indicating whether the crowdedness of the platform is "uncongested”, “congested (acceptable)".

From below table3, it can be seen that the passengers' perceived transfer waiting time is only affected by factors related to metro transfer that includes two influencing factors of the walking stage. This is indicating that the experiences and feelings of passengers during their walking journey will affect their waiting period. Additionally, the crowdedness of platforms is "uncongested" with an OR of 0.32, showing that compared to the situation of "very congested", the perceived transfer waiting time of passengers is generally lower when they are in an open and relaxed waiting environment. Finally, the cross terms of perceived transfer walking time and actual waiting time also play a significant role, indicating the passengers' feeling are related to both the actual situation of the current stage and the perception of the previous transfer stage.

Table 3 Estimation results of metro perceived transfer waiting time model

\begin{tabular}{ccccccc}
\hline Variable & Parameter & SD & Wald & Sig & \multicolumn{2}{c}{$\begin{array}{c}\text { 95\% Confidence interval } \\
\text { of the difference } \\
\text { Upper }\end{array}$} \\
& & & & & Lower \\
\hline$\alpha^{\prime}{ }_{1}$ & 2.68 & 0.77 & 12.21 & 0.00 & 1.18 & 4.18 \\
$\alpha^{\prime}{ }_{2}$ & 5.93 & 0.81 & 53.28 & 0.00 & 4.33 & 7.52 \\
$\mathrm{ZT}$ & 1.06 & 0.31 & 11.63 & 0.00 & 0.45 & 1.67 \\
$\mathrm{SD}_{1}$ & -0.81 & 0.38 & 4.49 & 0.03 & -1.56 & 0.06 \\
$\mathrm{SD}_{2}$ & -0.98 & 0.37 & 6.97 & 0.01 & -1.70 & 0.25 \\
$\mathrm{SD}_{3}$ & -0.65 & 0.38 & 2.88 & 0.09 & -1.41 & 0.10 \\
$\mathrm{ST}_{\mathrm{DR}}$ & 2.70 & 0.44 & 37.18 & 0.00 & 1.84 & 3.57 \\
$\mathrm{DR}_{2}$ & -1.13 & 0.38 & 8.78 & 0.00 & -1.87 & 0.38 \\
$\mathrm{ZT}$ & -0.21 & 0.21 & 1.06 & 0.30 & -0.62 & 0.19 \\
\hline
\end{tabular}

\subsection{Model Evaluation}

In order to evaluate the performance of the model, the global classification accuracy (GCA) 
representing the ratio of correctly classified instances, and the kappa coefficient are introduced. Additionally, several evaluation indicators are used to analyze the model's predictive power for different categories such as precision, recall and F-measure. Firstly, the GCA of metro perceived transfer time is $62.10 \%$ and of perceived transfer waiting time is $59.53 \%$ that both of them are over $50 \%$. Moreover, the kappa coefficient of metro perceived transfer time is 0.39 and of perceived transfer waiting time is 0.27 that both of values fall within the interval of 0.21 to 0.40 , showing the overall prediction ability is acceptable.

From below table 4 and table 5 , as to metro perceived transfer time, the category of " 5 minutes to 10 minutes (exclusive)" has the greatest predicted performance because it owns the highest value of recall and F-measure. For the metro perceived transfer waiting time, the category of " 3 minutes to 5 minutes (exclusive)" has the greatest predicted performance because of its highest value of recall, F-measure, and great value of precision. Moreover, the average value of F-measure of perceived transfer time is $52.18 \%$ and of perceived transfer waiting time is $54.16 \%$, meaning the latter has a more balanced predicting ability for different categories than the metro perceived transfer time model.

Table 4 Evaluation results of metro perceived transfer time model performance

\begin{tabular}{cccc}
\hline Metro perceived transfer time & Precision & Recall & F-measure \\
\hline Less than 5 minutes & 70.79 & 54.78 & 61.76 \\
5 minutes to 10 minutes (exclusive) & 60.35 & 79.63 & 68.66 \\
10 minutes to 15 minutes (exclusive) & 60.98 & 46.30 & 52.64 \\
15 minutes or more & 45.45 & 17.86 & 25.64 \\
\hline
\end{tabular}

Table 5 Evaluation results of metro perceived transfer waiting time model performance

\begin{tabular}{cccc}
\hline Metro perceived transfer waiting time & Precision & Recall & F-measure \\
\hline Less than 3 minutes & 55.87 & 57.80 & 56.82 \\
3 minutes to 5 minutes (exclusive) & 60.67 & 67.22 & 63.78 \\
5 minutes or more & 76.19 & 30.19 & 43.24 \\
\hline
\end{tabular}

\section{Scenario Analysis}

Based on the ordinal logistic regression model of metro perceived transfer time and of transfer waiting time, passengers' perceived transfer waiting time and transfer time will be predicted under different scenarios to explore the effect of changing the crowdedness of platform and the actual transfer waiting time on passengers' transfer time perception.

\subsection{Crowdedness of Platform}

Taking the samples with "very congested" as the study sample, passengers' perceived transfer waiting time and transfer time under the three scenarios are predicted, which scenario 1 is no change, scenario 2 changes to "congested (acceptable)", and scenario 3 changes to "uncongested". When the crowdedness of platform is changed from "very congested" to "congested (acceptable)" and then changed to "uncongested", the probability of perceived transfer waiting time of " 5 minutes or more" and "3 minutes to 5 minutes (exclusive)" decreases successively. Additionally, when it changes from "very congested" to "congested (acceptable)", the transfer time perception does not change, while it further becomes "uncongested", the transfer time perception of a few of passengers have decreased. This indicates that changing the crowdedness of platform can reduce the passenger's transfer waiting time perception to some extent, but it is difficult to change the transfer time perception. 
Table 6 Prediction results of perceived transfer waiting time (change the crowdedness of platform)

\begin{tabular}{cccc}
\hline Perceived transfer waiting time & Scenario 1 & Scenario 2 & Scenario 3 \\
\hline Less than 3 minutes & $24.14 \%$ & $31.80 \%$ & $51.72 \%$ \\
3minutes to 5 minutes (exclusive) & $68.58 \%$ & $62.84 \%$ & $48.28 \%$ \\
5 minutes or more & $7.28 \%$ & $5.36 \%$ & $0.00 \%$ \\
\hline
\end{tabular}

Table 7 Prediction results of perceived transfer time (change the crowdedness of platform)

\begin{tabular}{cccc}
\hline Transfer time perception & Scenario 1 & Scenario 2 & Scenario 3 \\
\hline Less than 5 minutes & $18.77 \%$ & $18.77 \%$ & $21.46 \%$ \\
5 minutes to 10 minutes (exclusive) & $64.37 \%$ & $64.37 \%$ & $61.30 \%$ \\
10 minutes to 15 minutes (exclusive) & $14.18 \%$ & $14.18 \%$ & $14.18 \%$ \\
15 minutes or more & $2.68 \%$ & $2.68 \%$ & $3.07 \%$ \\
\hline
\end{tabular}

\subsection{Actual Transfer Waiting Time}

Taking the sample of " 5 minutes or more" as the study sample, there is no change in scenario 1 and the actual waiting time is changed to " 3 minutes to 5 minutes (exclusive)" in scenario 2, and is changed to "less than 3 minutes" in scenario 3 . As can be seen from table 8 and table 9 that when the actual transfer waiting time changes from "5 minutes or more" to "3 minutes to 5 minutes (exclusive)", and then to "less than 3 minutes," passenger's perceived transfer waiting time and transfer time continues to decrease, but the degree of reduction is also in decline. In the first change, $84 \%$ passengers' perceived transfer time decrease, and 36\% passengers' perceived transfer waiting time reduce. However, in the second change, the reducing ratio of the perceived transfer time and the perceived transfer waiting time drop to $48 \%$ and $4 \%$. Therefore, it's cost-effective for both the operator and passengers when the actual transfer waiting time drops to "3 minutes to 5 minutes (exclusive)".

Table 8 Prediction results of perceived transfer waiting time (change the actual transfer waiting time)

\begin{tabular}{cccc}
\hline Perceived transfer waiting time & Scenario 1 & Scenario 2 & Scenario 3 \\
\hline Less than 3 minutes & $0.00 \%$ & $0.00 \%$ & $48.00 \%$ \\
3minutes to 5 minutes (exclusive) & $16.00 \%$ & $100.00 \%$ & $52.00 \%$ \\
5 minutes or more & $84.00 \%$ & $0.00 \%$ & $0.00 \%$ \\
\hline
\end{tabular}

Table 9 Prediction results of perceived transfer time (change the actual transfer waiting time)

\begin{tabular}{cccc}
\hline Transfer perception time & Scenario 1 & Scenario 2 & Scenario 3 \\
\hline Less than 5 minutes & $4.00 \%$ & $40.00 \%$ & $44.00 \%$ \\
5 minutes to 10 minutes (exclusive) & $88.00 \%$ & $60.00 \%$ & $56.00 \%$ \\
10 minutes to 15 minutes (exclusive) & $8.00 \%$ & $0.00 \%$ & $0.00 \%$ \\
15 minutes or more & $0.00 \%$ & $0.00 \%$ & $0.00 \%$ \\
\hline
\end{tabular}

\section{Conclusion}

This study verifies the passengers' perceived difference in their transfer phase by paired-sample T test, which is reflected in the overestimation of perception of transfer time and transfer distance. Moreover, the results of model evaluation reveal that the model of perceived transfer time and of transfer waiting time proposed in this study can effectively predict the passenger's transfer time perception and transfer waiting time perception. Finally, the study provides some strategic guidance for reducing passengers' perceived transfer time through scenario analysis. The results of the scenario analysis show that reducing the actual waiting time is obviously more effective than changing the 
crowdedness of platform, because passengers' transfer time perception drops significantly as the actual transfer waiting time decreases, especially when the actual transfer waiting time is reduced to “3 minutes to 5 minutes".

\section{Acknowledgements}

This study is supported by National Natural Science Foundation of China [grant number 71571011] and the Fundamental Research Funds for the Central Universities [grant number 2018JBM022].

\section{References}

[1] Yu M. A Study of the travel perception time of urban public transportation. Southeast University, China, 2008.

[2] Delclòs-Alió X, Marquet O, Miralles-Guasch C. 2017. Keeping track of time: A Smartphone-based analysis of travel time perception in a suburban environment. Travel Behavior and Society. Vol. 9.

[3] Zhu J. The optimization on study of transit station spacing base on passengers' perceived travel time. Southwest Jiaotong University, China, 2017.

[4] Watkins K E, Ferris B, Borning A, et al. 2011. Where Is My Bus? Impact of mobile real-time information on the perceived and actual wait time of transit riders. Transportation Research Part A: Policy and Practice. Vol. 45(8).

[5] Fan Y, Guthrie A, Levinson D. 2016. Waiting time perceptions at transit stops and stations: Effects of basic amenities, gender, and security. Transportation Research Part A: Policy and Practice. Vol. 88.

[6] González R M, Martínez-Budría E, Díaz-Hernández J J, et al. 2015. Explanatory factors of distorted perceptions of travel time in tram. Transportation Research Part F: Traffic Psychology and Behaviour. Vol. 30.

[7] Meng M, Rau A, Mahardhika H. 2018. Public transport travel time perception: Effects of socioeconomic characteristics, trip characteristics and facility usage. Transportation Research Part A: Policy and Practice. Vol. 114.

[8] Zhao L, Bian Y, Rong J, et al. 2014. Pedestrian LOS of Urban Sidewalks Based on Orderly Logistic Regression. Journal of Transportation Systems Engineering and Information Technology. Vol. 14(4).

[9] Zheng Z, Lee J, Saifuzzaman M, et al. 2015. Exploring association between perceived importance of travel/traffic information and travel behavior in natural disasters: A case study of the 2011 Brisbane floods. Transportation Research Part C: Emerging Technologies. Vol. 51.

[10] Xie W. SPSS Statistical Analysis Method and Application. Publishing House of Electronics Industry, China, 2017. 